\title{
Economic Value of Dispensing Home-Based Preoperative Chlorhexidine Bathing Cloths to Prevent Surgical Site Infection
}

\author{
Rachel R. Bailey, PhD, MPH 1,2,3, Dianna R. Stuckey ${ }^{1,2,3}$, Bryan A. Norman, PhD $^{4}$, Andrew P. \\ Duggan $^{4}$, Kristina M. Bacon, MPH ${ }^{1,2,3}$, Diana L. Connor, $\mathbf{M P H}^{1,2,3}$, Ingi Lee, MD, MSCE ${ }^{5,6}$, \\ Robert R. Muder, $\mathbf{M D}^{7}$, and Bruce Y. Lee, MD, MBA ${ }^{1,2,3}$ \\ ${ }_{1}^{1}$ Public Health Computational and Operations Research Group, University of Pittsburgh, \\ Pittsburgh, Pennsylvania \\ ${ }^{2}$ Department of Biomedical Informatics, University of Pittsburgh, Pittsburgh, Pennsylvania \\ ${ }^{3}$ Department of Epidemiology, University of Pittsburgh, Pittsburgh, Pennsylvania \\ ${ }^{4}$ Department of Industrial Engineering, University of Pittsburgh, Pittsburgh, Pennsylvania \\ ${ }^{5}$ Division of Infectious Diseases, Department of Medicine, University of Pennsylvania, \\ Philadelphia, Pennsylvania \\ ${ }^{6}$ Center for Evidence-Based Practice of the University of Pennsylvania School of Medicine, \\ Philadelphia, Pennsylvania \\ ${ }^{7}$ Division of Infectious Diseases, Veterans Affairs Pittsburgh Healthcare System, Pittsburgh, \\ Pennsylvania
}

\section{Abstract}

OBJECTIVE-To estimate the economic value of dispensing preoperative home-based chlorhexidine bathing cloth kits to orthopedic patients to prevent surgical site infection (SSI).

METHODS-A stochastic decision-analytic computer simulation model was developed from the hospital's perspective depicting the decision of whether to dispense the kits preoperatively to orthopedic patients. We varied patient age, cloth cost, SSI-attributable excess length of stay, cost per bed-day, patient compliance with the regimen, and cloth antimicrobial efficacy to determine which variables were the most significant drivers of the model's outcomes.

\begin{abstract}
RESULTS-When all other variables remained at baseline and cloth efficacy was at least 50\%, patient compliance only had to be half of baseline (baseline mean, 15.3\%; range, 8.23\%-20.0\%) for chlorhexidine cloths to remain the dominant strategy (ie, less costly and providing better health outcomes). When cloth efficacy fell to $10 \%, 1.5$ times the baseline bathing compliance also afforded dominance of the preoperative bath.
\end{abstract}

CONCLUSIONS-The results of our study favor the routine distribution of bathing kits. Even with low patient compliance and cloth efficacy values, distribution of bathing kits is an economically beneficial strategy for the prevention of SSI.

Surgical site infection (SSI) is a substantial problem in the United States. Approximately 46 million surgeries are performed annually, and at least 1 in every 100 procedures is

(C) 2011 by The Society for Healthcare Epidemiology of America. All rights reserved.

Address correspondence to Rachel R. Bailey, MPH, Public Health Computational and Operations Research (PHICOR), University of Pittsburgh, 3520 Forbes Avenue, First Floor, Pittsburgh, PA 15213 (RRB16@ pitt.edu).

Potential conflicts of interest. All authors report no conflicts of interest relevant to this article. 
complicated by an SSI during hospitalization. ${ }^{1}$ These infections are often associated with higher morbidity and mortality rates as well as increased lengths of stay (LOS). In 2002, there were approximately 1.7 million SSI cases, resulting in 99,000 deaths. $^{2}$ The economic burden associated with these preventable morbidity and mortality rates is high, and a reduction in these values would be advantageous for healthcare facilities. ${ }^{2,3}$ It is increasingly important that hospitals begin to adopt preventive measures to increase the safety of their patients and reduce the high costs associated with these infections.

Antiseptic bathing is one of the preoperative procedures recommended by the Centers for Disease Control and Prevention. ${ }^{4}$ Previous studies have shown that screening surgical patients for Staphylococcus aureus infection and selectively de-colonizing those who test positive with a regimen including chlorhexidine baths is a cost-effective strategy. 5,6 However, whether to routinely provide preoperative antiseptic bathing to all patients remains unclear. Low patient compliance rates coupled with varying antimicrobial efficacy reported in recent studies have limited adoption of this prevention technique (A. Johnson, written personal communication, May 2010). ${ }^{7-10}$

Our study focuses on the use of home-based patient-applied chlorhexidine cloths because recent studies have shown chlorhexidine to be the optimal antiseptic agent for the preoperative bathing of orthopedic patients. ${ }^{9,10}$ Unlike other available antiseptic agents (ie, alcohol and povidone-iodine), chlorhexidine is relatively odorless and colorless, which results in higher observed compliance values. It is also not flammable, making it safer for use in the operating room, and it exhibits greater antibacterial power. ${ }^{4,11-14}$ Preoperative chlorhexidine rinse is available both as a liquid soap and as a saturated polyester cloth, with recent studies noting increased use of the polyester cloth compared with the liquid soap. Despite past reviews, which have deemed chlorhexidine bathing to be an unnecessary preoperative procedure, results of recent clinical trials have been favorable (A. Johnson, written personal communication, May 2010). ${ }^{7-10}$

We designed a computer simulation model to determine from the perspective of the hospital the economic value of preoperative chlorhexidine bathing for orthopedic patients with polyester cloths. A variety of sensitivity analyses evaluated how varying patient compliance, patient age, chlorhexidine cloth efficacy (ie, the accompanying decrease in the probability of postoperative SSI with preoperative home-based bathing), excess LOS attributable to SSI, and costs influence the cost-effectiveness of the bathing strategy.

\section{METHODS}

Using TreeAge Pro 2009 (TreeAge Software), we developed a stochastic decision-analytic computer simulation model depicting the decision of whether to distribute a chlorhexidine cloth kit to patients for home-based preoperative bathing (Figure 1) in addition to standard in-hospital preoperative preparation. The model evaluated the effects of the distribution of preoperative chlorhexidine bathing kits for the prevention of SSI in patients undergoing orthopedic (hip and knee) surgery. Preoperative bathing refers to the application of the chlorhexidine cloths the evening before and the morning of the surgical procedure. Each kit contains 12 cloths -6 cloths for 2 preoperative baths that include the disinfection of a patient's head, abdomen, arms, legs, back, and surgical site. ${ }^{9,10}$ In-hospital preoperative procedures included pre-operative antibiotic prophylaxis with infusion beginning 60 minutes before incision of 1-2 $\mathrm{g}$ of intravenous cefazolin, $1.5 \mathrm{~g}$ of intravenous cefuroxime, or $1 \mathrm{~g}$ of intravenous vancomycin. Additionally, surgical site preparation was done with a combination iodine poyacrylaex-alcohol preparation (DuraPrep solution; $3 \mathrm{M}$ ) and was standardized among the intervention and no-intervention groups. ${ }^{9,10}$ Postoperative 
procedures were also standardized in both study populations and have been described in detail elsewhere. ${ }^{9,10}$

The model assumed the hospital perspective (ie, the costs and health benefits experienced by the hospital) and simulated the possible cost-effectiveness of chlorhexidine cloth use under a range of conditions (varying patient age, cloth cost, cost per bed-day, SSI-attributable excess LOS, patient compliance, and cloth antimicrobial efficacy). The hospital perspective includes only the inpatient costs associated with an SSI, including excess LOS, use of antibiotics, isolation expenses, and physician and nurse time. Lost wages, travel expenses, and outpatient treatments are not included in analyses from the hospital perspective. ${ }^{15}$ Outcomes were dependent on the increased LOS for patients who acquired an SSI and included only costs associated with this increase to provide an appropriate monetary valuation of the cost of SSIs. ${ }^{1}$ A patient's risk of infection after surgery was dependent on SSI risk, cloth efficacy, and compliance data compiled from 2 similar studies (A. Johnson, written personal communication, May 2010). 9,10

Each simulation run sent 1,000 orthopedic patients through the model 1,000 times, for a total of 1,000,000 trials. Each patient was 63 years of age and healthy when entering the model. As each patient traveled through the model, he or she had the chance of accumulating costs and quality-adjusted life-years (QALYs) associated with the path he or she traveled. A healthy individual aged 0-17 years accrues 1 QALY per year. Individuals aged 18-64 years can accrue a maximum of 0.92 QALYs during a healthy year, whereas those 65 years old or more accrue 0.84 QALYs during a healthy year. ${ }^{11}$ After all patients were run through the model, the costs and QALY values of the 1,000,000 trials were combined, and an average was computed for the simulation.

For each simulation run, an incremental cost-effectiveness ratio (ICER) was used to calculate the added cost of maintaining a QALY. The following equation calculates the ICER of using chlorhexidine cloths as a preoperative technique to prevent SSIs:

$$
\operatorname{ICER}=\left(C_{\text {cloth }}-C_{\text {no cloth }}\right) /\left(E_{\text {cloth }}-E_{\text {no cloth }}\right),
$$

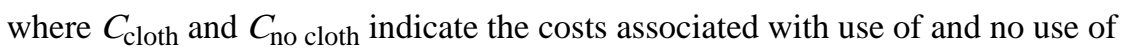
chlorhexidine cloths, respectively, and $E_{\text {cloth }}$ and $E_{\text {no cloth }}$ indicate the effectiveness associated with use of and no use of chlorhexidine cloths, respectively.

ICER values below $\$ 50,000$ per QALY were considered relatively cost-effective, and those equal to or above the threshold were considered not cost-effective. ${ }^{16}$ When the preoperative chlorhexidine cloth intervention is both less costly and provides better health outcomes than no cloth intervention, it is known as the dominant strategy-that is, there is no cost or health effect disadvantage in implementation. Likewise, when the pre-operative chlorhexidine cloth intervention is more costly and less effective than no cloth intervention, it is considered the dominated approach, and implementation is discouraged.

Table 1 lists the costs, probabilities, time intervals, and effectiveness values used as inputs for our preoperative chlorhexidine bath model as well as corresponding distributions and data sources. All input parameters assume triangular distributions except the cost of the chlorhexidine cloths used for bathing, which assumes a gamma distribution. Gamma distributions are used to account for variables with skewed distributions, such as costs. ${ }^{17}$ Triangular distributions are used when existing data are limited, such as skewed confidence intervals with an upper and lower bound, and resemble the shape of a triangle. Using International Classification of Diseases, Ninth Revision, code 81.54 for total knee replacement, hospital costs were extracted from the Healthcare Cost and Utilization 
Project's National Inpatient Survey; the cost per bed-day was $\$ 4,771 .{ }^{18}$ Cost of the chlorhexidine cloths was systematically determined using various online sources from which a mean and standard deviation were calculated. A 3\% discount rate was used to convert all costs into 2010 US dollars. ${ }^{19}$

Our model measured the effectiveness of bathing with a chlorhexidine cloth in QALYs. Each medical condition caused a QALY decrement, which endured only for the duration of the ailment. Most patients undergoing an orthopedic procedure have an age-adjusted baseline QALY value of 0.84 as a result of older age. After the procedure, patients with an SSI have a QALY value of 0.756, accounting for the decreased quality of life for the duration of an SSI. Because all patients traveling through the model undergo the same surgical procedure, the associated QALY decrement value for orthopedic surgical patients was null and would not have affected the model's outcomes. ${ }^{11,20}$ SSIs have a mean duration of 9.5 days, so QALY values represented this fraction of the whole year. The time horizon (or period of time included in each simulation) was 1 year.

Sensitivity analyses were conducted to determine the effect that varying efficacies and costs would have on the cost-effectiveness of the preoperative procedure. We systematically tested a wide range of chlorhexidine bath efficacies $(10 \%, 25 \%, 50 \%$, and $75 \%)$ and patient compliance rates ( 0.25 times baseline compliance to 2.0 times baseline compliance) to evaluate variations from the baseline data found in Table 1 (mean compliance, 15.3\%; range, $8.32 \%-20.0 \%$ ). We ran additional simulations varying patient age (53-63 years old), chlorhexidine cloth costs (\$10.00-\$100.00), SSI-attributable excess LOS (5-15 days), and cost per bed-day $(\$ 3,000-\$ 10,000)$.

\section{RESULTS}

When the model was run at the baseline cost scenario (mean cost, \$29.35; standard deviation, \$7.89), distribution of pre-operative bathing kits was the economically dominant strategy when cloth efficacy was at least $10 \%$ and compliance was at least 1.5 times the baseline distribution, when cloth efficacy was at least $25 \%$ and compliance was at least $75 \%$ of baseline, and when cloth efficacy was at least $50 \%$ and compliance was at least $50 \%$ of baseline. Chlorhexidine cloth cost, excess LOS, patient compliance rates, and cloth efficacy were the most significant drivers of the model's outcomes. Patient age (53-63 years old) and cost per bed-day $(\$ 3,000-\$ 10,000)$ did not substantially affect model outcomes and are not presented.

\section{Cost of Chlorhexidine Cloths}

Table 2 shows the cost results from our analysis. As the cost of the chlorhexidine cloths was increased from the baseline value to $\$ 100$, the preoperative cloth became highly costineffective at most compliance values and cloth efficacies. At this relatively high cost, distributing the preoperative bathing kits was the dominant strategy only when cloth efficacy was at least $75 \%$ and patient compliance was at least $75 \%$ of baseline (mean, $11.48 \%$ ), when cloth efficacy was at least 50\% and compliance was at least the baseline distribution (mean, $15.3 \%$; range, $8.23 \%-20.0 \%$ ), and when cloth efficacy was at least $25 \%$ and compliance was twice the baseline rate (mean, 30.6\%). When the cost of the cloths was decreased to $\$ 10$ from the baseline rate of $\$ 29.35$ (standard deviation, $\$ 7.89$ ), preoperative bathing became the dominant strategy for all scenarios with efficacy at least $10 \%$ and compliance at least $50 \%$ of the baseline (mean, $7.65 \%$ ). Results for an increase in cloth cost to $\$ 50$ varied little from baseline results. 


\section{Excess LOS Attributable to SSI}

Table 3 shows the LOS results from our analysis. As the excess LOS attributable to SSI for patients was increased from the baseline value of 9.5 days (range, 7.7-11.7 days) to 15 days, the distribution of preoperative bathing kits became dominant at all patient compliance rates when cloth efficacy was $25 \%$ or more. When the duration of excess LOS was equal to 15 days and cloth efficacy was $10 \%$, the bathing strategy was dominant as long as patient compliance was at least $75 \%$ of the baseline value (mean, $11.48 \%$ ). When the excess LOS for patients was decreased from the baseline value (mean, 9.5 days) to 5 days, the kit distribution became dominant in fewer scenarios, specifically at $25 \%$ cloth efficacy. Extending the excess LOS from the baseline value to 15 days seemed to have a greater effect on the model's outcomes than did decreasing the LOS.

\section{Patient Compliance with Bathing}

Although cost and LOS affected the model's outcomes, patient compliance rates had the greatest effect on results. At baseline compliance (mean, 15.3\%; range, 8.23\%-20.0\%), the chlorhexidine cloth was dominant in all simulated runs as long as chlorhexidine cloth efficacy was at least $50 \%$. When patient compliance values were reduced to at least $50 \%$ of baseline (mean, $7.65 \%$ ) and chlorhexidine cloth costs were no greater than $\$ 50$, the chlorhexidine bathing strategy was dominant, as long as the chlorhexidine cloth efficacy was $50 \%$. Even when patient compliance was lower than $50 \%$ of baseline (mean, less than 7.65\%), the bathing strategy continued to be the dominant strategy at high cloth efficacy. When patient compliance was doubled, the chlorhexidine bath strategy was dominant in the simulated runs across the range of sensitivity analysis values as long as chlorhexidine bath efficacy was at least $25 \%$.

\section{Avoided Infections}

Figure 2 presents a graphical representation of the number of infections avoided per million preoperative patients to whom the cloths are distributed. At baseline patient compliance (mean, 15.3\%; range, $8.23 \%-20.0 \%$ ) and 25\% chlorhexidine cloth efficacy, 1,283 SSIs were avoided per million kits distributed. This increased to 2,258 SSIs avoided at 50\% efficacy. When compliance was half of baseline compliance (mean, 7.65\%), the number of SSIs avoided dropped to 1,225 at a cloth efficacy of $50 \%$. When patient compliance was double the baseline value and cloth efficacy remained at 50\%, the number of infections avoided climbed to 4,724 .

\section{Bath Interventions}

Table 4 shows the number of bathing kits that need to be dispensed to prevent 1 SSI, and Figure 3 presents a graphical representation of the data. The number of kits that need to be distributed to prevent 1 SSI was calculated when cost and LOS was held at baseline, which for cost was $\$ 29.35$ ( $\pm \$ 7.89$ ) and for LOS was 9.5 days (range, 7.7-11.7 days). As patient compliance increased, the absolute number of patients who needed to be prescribed a chlorhexidine bathing kit decreased. When compliance was a quarter of baseline (mean, $3.83 \%$ ), the difference in the number of bathing interventions was 5,841. When compliance was doubled from baseline (mean, 40\%), the difference dropped to 678 .

\section{DISCUSSION}

Our model demonstrates that home-based preoperative bathing with chlorhexidineimpregnated cloths is a cost-effective strategy across a wide range of antimicrobial efficacy and patient compliance values. The intervention remains cost-effective even for fairly low cloth efficacies and patient compliance values. For bathing to remain the economically 
dominant strategy, cloth efficacy can be as low as $10 \%$ as long as patient compliance is doubled from baseline to a mean value from a previous study of $30.60 \%$ (range, $16.46 \%-$ $40.00 \%$ ). Conversely, patient compliance can be as low as half of the baseline value (mean, $7.65 \%$; range, $4.16 \%-10.00 \%$ ) as long as cloth efficacy was $75 \%$. The patient compliance and cloth efficacy pair was the impetus of the economic value of pre-operative chlorhexidine bathing.

To date, most of the opposition to home-based preoperative chlorhexidine bathing has emerged from concerns about low cloth antimicrobial efficacy and patient compliance. However, our study suggests that these concerns may not be as crucial as initially thought. Although the Cochrane Collaboration's 2009 systematic review assessing the effectiveness of preoperative bathing suggested that preoperative bathing with chlorhexidine was not an effective measure in the prevention of SSI, this review did not include studies using a relatively new chlorhexidine cloth application. ${ }^{7,10}$ The non-woven polyester fiber cloth, unlike traditional cotton wash-cloths, results in a higher skin surface concentration of chlorhexidine associated with a greater antimicrobial effect. ${ }^{8}$ Because our study assumed that patients would receive other standard SSI prevention (e.g., prophylactic antibiotics, proper hair removal, and surgical site preparation), our results suggest that there is additional value in including the distribution of preoperative home-based bathing kits in a suite of surgical site prevention techniques used by surgeons.

Limitations of our study include the following. All computer simulation models are simplifications of real-life scenarios and cannot represent all possible situations and outcomes. Our data inputs came from existing literature that explored the implementation of chlorhexidine cloths as an SSI prevention technique, and sensitivity analyses were performed to assess the robustness of the results. Additional clinical studies may further explore the effect of home-based preoperative chlorhexidine bathing and could be used to update our model in the future.

Controversy remains over the routine dispensing of home-based preoperative chlorhexidine bathing kits because of concerns over antimicrobial efficacy and patient compliance. However, our model suggests that such a strategy would have substantial economic value across a wide range of patient compliance levels, cloth efficacies, cloth costs, and SSIattributable LOS. Our study supports the distribution of chlorhexidine cloths for preoperative bathing because this intervention remains cost-effective over a wide range of cloth efficacy and patient compliance values.

\section{Acknowledgments}

Financial support. This study was supported by the National Institute of General Medical Sciences Models of Infectious Disease Agent Study (grant 1U54GM088491-0109) and the Pennsylvania Department of Health (grant 4100047864).

\section{References}

1. Graves N, Harbarth S, Beyersmann J, Barnett A, Halton K, Cooper B. Estimating the cost of health care-associated infections: mind your p's and q's. Clin Infect Dis. 2010; 50(7):1017-1021. [PubMed: 20178419]

2. de Lissovoy G, Fraeman K, Hutchins V, Murphy D, Song D, Vaughn BB. Surgical site infection: incidence and impact on hospital utilization and treatment costs. Am J Infect Control. 2009; 37(5): 387-397. [PubMed: 19398246]

3. Evans RP. Surgical site infection prevention and control: an emerging paradigm. J Bone Joint Surg Am. 2009; 91(suppl 6):2-9. [PubMed: 19884406] 
4. Mangram AJ, Horan TC, Pearson ML, Silver LC, Jarvis WR. Hospital Infection Control Practices Advisory Committee. Guideline for prevention of surgical site infection, 1999. Infect Control Hosp Epidemiol. 1999; 20(4):250-278. [PubMed: 10219875]

5. Lee BY, Tsui BY, Bailey RR, et al. Should vascular surgery patients be screened preoperatively for methicillin-resistant Staphylococcus aureus? Infect Control Hosp Epidemiol. 2009; 30(12):11581165. [PubMed: 19852665]

6. Lee BY, Wiringa AE, Bailey RR, et al. Screening cardiac surgery patients for MRSA: an economic computer model. Am J Manag Care. 2010; 16(7):e163-e173. [PubMed: 20645662]

7. Webster J, Osborne S. Preoperative bathing or showering with skin antiseptics to prevent surgical site infection. Cochrane Database Syst Rev. 2007; (2):CD004985. [PubMed: 17443562]

8. Edmiston CE Jr, Krepel CJ, Seabrook GR, Lewis BD, Brown KR, Towne JB. Preoperative shower revisited: can high topical antiseptic levels be achieved on the skin surface before surgical admission? J Am Coll Surg. 2008; 207(2):233-239. [PubMed: 18656052]

9. Johnson AJ, Daley JA, Zywiel MG, Delanois RE, Mont MA. Preoperative chlorhexidine preparation and the incidence of surgical site infections after hip arthroplasty. J Arthroplasty. 2010; 25(6 suppl 1):98-102. [PubMed: 20570089]

10. Zywiel MG, Daley JA, Delanois RE, Naziri Q, Johnson AJ, Mont MA. Advance pre-operative chlorhexidine reduces the incidence of surgical site infections in knee arthroplasty. Int Orthop. Published June 20, 2010. 10.1007/s00264-010-1078-5

11. Gold MR, Franks P, McCoy KI, Fryback DG. Toward consistency in cost-utility analyses: using national measures to create condition-specific values. Med Care. 1998; 36(6):778-792. [PubMed: 9630120]

12. Kampf G, Kramer A. Epidemiologic background of hand hygiene and evaluation of the most important agents for scrubs and rubs. Clin Microbiol Rev. 2004; 17(4):863-893. [PubMed: 15489352]

13. Garibaldi RA. Prevention of intraoperative wound contamination with chlorhexidine shower and scrub. J Hosp Infect. 1988; 11(suppl B):5-9. [PubMed: 2898503]

14. Lee I, Agarwal RK, Lee BY, Fishman NO, Umscheid CA. Systematic review and cost analysis comparing use of chlorhexidine with use of iodine for preoperative skin antisepsis to prevent surgical site infection. Infect Control Hosp Epidemiol. 2010; 31(12):1219-1229. [PubMed: 20969449]

15. Maragakis LL, Perencevich EN, Cosgrove SE. Clinical and economic burden of antimicrobial resistance. Expert Rev Anti Infect Ther. 2008; 6(5):751-763. [PubMed: 18847410]

16. Laupacis A, Feeny D, Detsky AS, Tugwell PX. How attractive does a new technology have to be to warrant adoption and utilization? tentative guidelines for using clinical and economic evaluations. CMAJ. 1992; 146(4):473-481. [PubMed: 1306034]

17. Thompson SG, Nixon RM. How sensitive are cost-effectiveness analyses to choice of parametric distributions? Med Decis Making. 2005; 25(4):416-423. [PubMed: 16061893]

18. Farley JE, Stamper PD, Ross T, Cai M, Speser S, Carroll KC. Comparison of the BD GeneOhm methicillin-resistant Staphylococcus aureus (MRSA) PCR assay to culture by use of BBL CHROMagar MRSA for detection of MRSA in nasal surveillance cultures from an at-risk community population. J Clin Microbiol. 2008; 46(2):743-746. [PubMed: 18057129]

19. Shepard, DS. Cost-effectiveness in health and medicine. In: Gold, MR.; Siegel, JE.; Russell, LB.; Weinstein, MC., editors. J Ment Health Policy Econ. Vol. 2. New York: Oxford University Press; 1999. p. 91-92.

20. Tengs TO, Wallace A. One thousand health-related quality-of-life estimates. Med Care. 2000; 38(6):583-637. [PubMed: 10843310]

21. Pereira BJ, Shapiro L, King AJ, Falagas ME, Strom JA, Dinarello CA. Plasma levels of IL-1 beta, TNF alpha and their specific inhibitors in undialyzed chronic renal failure, CAPD and hemodialysis patients. Kidney Int. 1994; 45(3):890-896. [PubMed: 8196293]

22. Zeltzer E, Bernheim J, Korzets Z, et al. Diminished chemokine and cytokine-induced adhesion of $\mathrm{CD} 4+\mathrm{T}$ cells to extracellular matrix ligands in patients with end-stage renal failure. Isr Med Assoc J. 2000; 2(4):282-286. [PubMed: 10804903] 
23. Anandh U, Bastani B, Ballal S. Granulocyte-macrophage colony-stimulating factor as an adjuvant to hepatitis B vaccination in maintenance hemodialysis patients. Am J Nephrol. 2000; 20(1):5356. [PubMed: 10644869]

24. Fabrizi F, Ganeshan SV, Dixit V, Martin P. Meta-analysis: the adjuvant role of granulocyte macrophage-colony stimulating factor on immunological response to hepatitis B virus vaccine in end-stage renal disease. Aliment Pharmacol Ther. 2006; 24(5):789-796. [PubMed: 16918882]

25. Jha R, Lakhtakia S, Jaleel MA, Narayan G, Hemlatha K. Granulocyte macrophage colony stimulating factor (GM-CSF) induced sero-protection in end stage renal failure patients to hepatitis B in vaccine non-responders. Ren Fail. 2001; 23(5):629-636. [PubMed: 11725909]

26. Singh NP, Mandal SK, Thakur A, et al. Efficacy of GM-CSF as an adjuvant to hepatitis B vaccination in patients with chronic renal failure-results of a prospective, randomized trial. Ren Fail. 2003; 25(2):255-266. [PubMed: 12739832]

27. Sudhagar K, Chandrasekar S, Rao MS, Ravichandran R. Effect of granulocyte macrophage colony stimulating factor on hepatitis-B vaccination in haemodialysis patients. J Assoc Physicians India. 1999; 47(6):602-604. [PubMed: 10999156] 


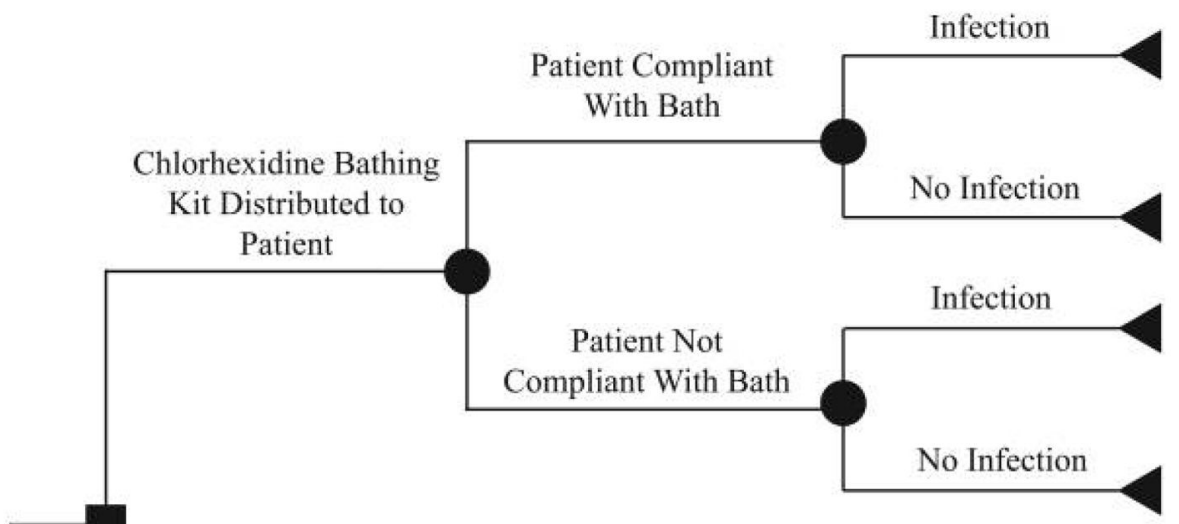

FIGURE 1.

Model structure for deciding whether to distribute chlorhexidine bathing cloth kits to study patients. 


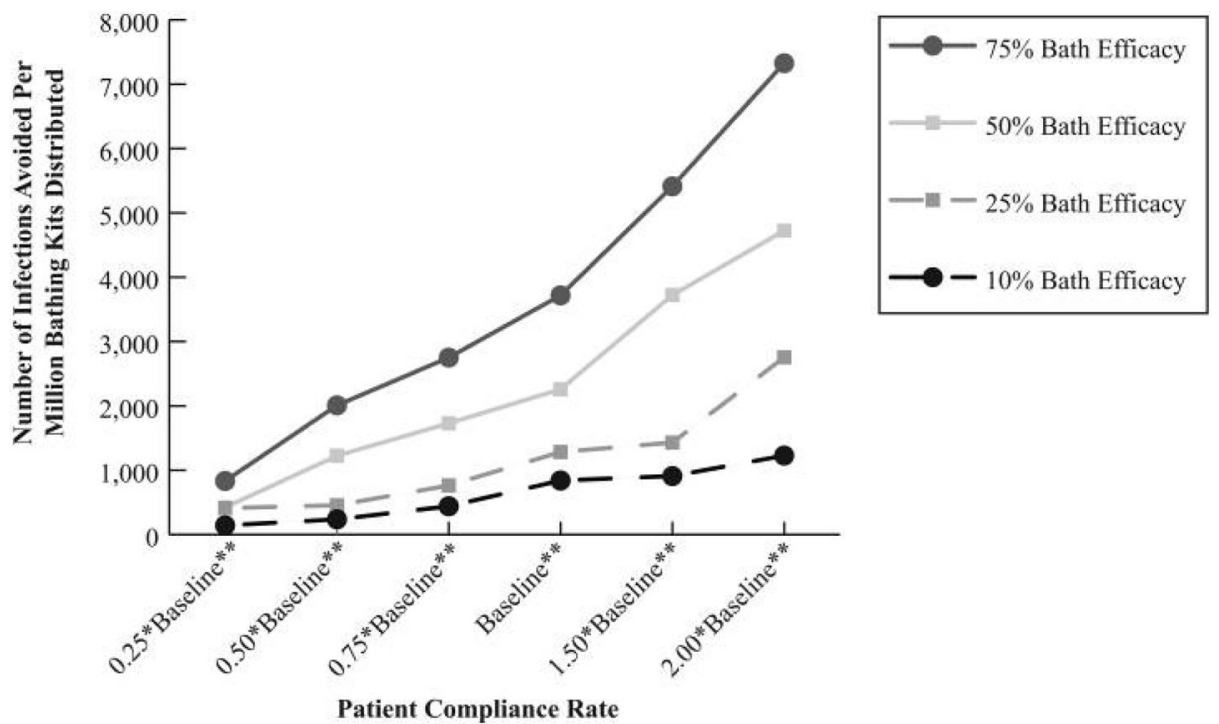

FIGURE 2.

Patient compliance rate versus number of infections avoided per million bathing kits distributed. Two asterisks indicate that the baseline data have a triangular distribution with a mean value of $15.3 \%$ and a range of $8.23 \%-20.0 \%$. 


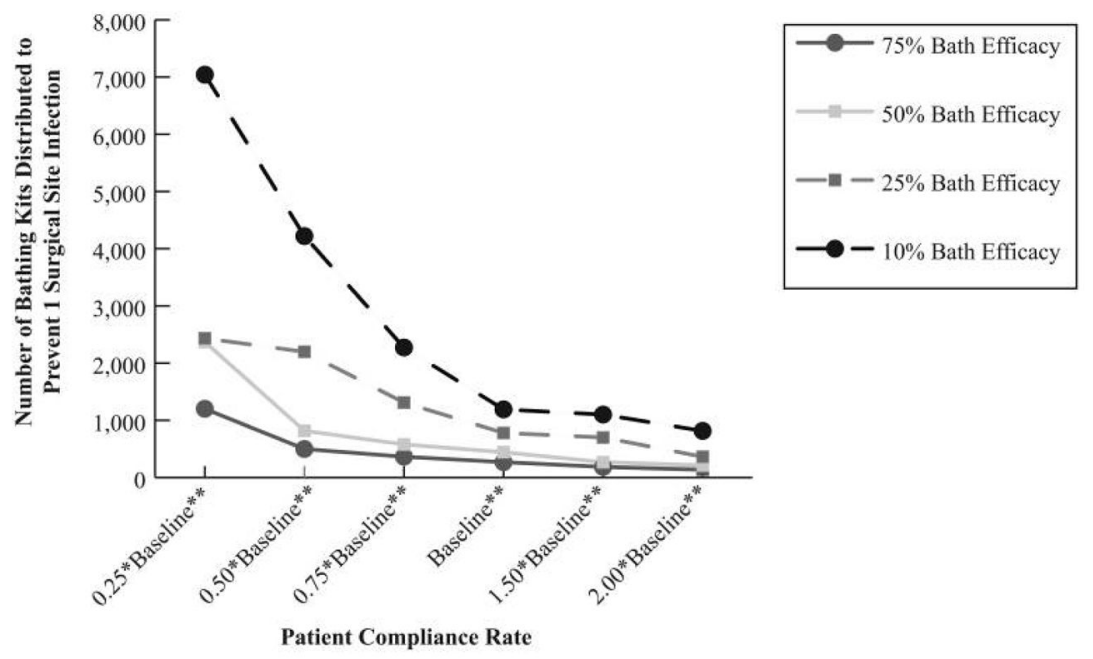

FIGURE 3.

Patient compliance rate versus number of bathing kits distributed to prevent 1 surgical site infection. Two asterisks indicate that the baseline data have a triangular distribution with a mean value of $15.3 \%$ and a range of $8.23 \%-20.0 \%$. 


\section{TABLE 1}

Model Input Parameters

\begin{tabular}{lcc}
\hline Description & Value & Reference(s) \\
\hline Cost, \$US & $29.35 \pm 7.89$ & $21-27$ \\
$\quad$ Chlorhexidine wipes & & \\
Probabilities & $0.153(0.0823-0.200)$ & 9,10 \\
Compliance & $0.025(0.008-0.065)$ & 9,10 \\
SSI without bath & & 2 \\
Time, days & $9.5(7.7-11.7)$ & 11 \\
Infection-attributable excess LOS & & 20 \\
Effectiveness & 0.84 & \\
Age 63 years QALY value & 0.90 & \\
Orthopedic SSI QALY value & & \\
\hline
\end{tabular}

NOTE. Data are mean \pm standard deviation or mean (range). LOS, length of stay; SSI, surgical site infection; QALY, quality-adjusted life-year. 
TABLE 2

Model Predictions of the Cost-Effectiveness of Chlorhexidine Baths on the Basis of Cloth Cost

\begin{tabular}{|c|c|c|c|c|}
\hline \multirow[b]{2}{*}{ Cloth cost, compliance rate ${ }^{a}$} & \multicolumn{4}{|c|}{ Bath efficacy in preventing SSIs } \\
\hline & $10 \%$ & $25 \%$ & $50 \%$ & $75 \%$ \\
\hline \multicolumn{5}{|l|}{$\$ 10.00$} \\
\hline One-fourth of baseline compliance & $>1$ million & Dominant & Dominant & Dominant \\
\hline One-half of baseline compliance & Dominant & Dominant & Dominant & Dominant \\
\hline Three-fourths of baseline compliance & Dominant & Dominant & Dominant & Dominant \\
\hline Baseline compliance & Dominant & Dominant & Dominant & Dominant \\
\hline 1.5 times baseline compliance & Dominant & Dominant & Dominant & Dominant \\
\hline 2.0 times baseline compliance & Dominant & Dominant & Dominant & Dominant \\
\hline \multicolumn{5}{|l|}{ Baseline cost } \\
\hline One-fourth of baseline compliance & $>1$ million & $>1$ million & $>1$ million & $>1$ million \\
\hline One-half of baseline compliance & $>1$ million & $>1$ million & Dominant & Dominant \\
\hline Three-fourths of baseline compliance & $>1$ million & Dominant & Dominant & Dominant \\
\hline Baseline compliance & $>1$ million & Dominant & Dominant & Dominant \\
\hline 1.5 times baseline compliance & Dominant & Dominant & Dominant & Dominant \\
\hline 2.0 times baseline compliance & Dominant & Dominant & Dominant & Dominant \\
\hline \multicolumn{5}{|l|}{$\$ 50.00$} \\
\hline One-fourth of baseline compliance & $>1$ million & $>1$ million & $>1$ million & $>1$ million \\
\hline One-half of baseline compliance & $>1$ million & $>1$ million & Dominant & Dominant \\
\hline Three-fourths of baseline compliance & $>1$ million & $>1$ million & Dominant & Dominant \\
\hline Baseline compliance & $>1$ million & Dominant & Dominant & Dominant \\
\hline 1.5 times baseline compliance & $>1$ million & Dominant & Dominant & Dominant \\
\hline 2.0 times baseline compliance & $>1$ million & Dominant & Dominant & Dominant \\
\hline \multicolumn{5}{|l|}{$\$ 100.00$} \\
\hline One-fourth of baseline compliance & $>1$ million & $>1$ million & $>1$ million & $>1$ million \\
\hline One-half of baseline compliance & $>1$ million & $>1$ million & $>1$ million & $>1$ million \\
\hline Three-fourths of baseline compliance & $>1$ million & $>1$ million & $>1$ million & Dominant \\
\hline Baseline compliance & $>1$ million & $>1$ million & Dominant & Dominant \\
\hline 1.5 times baseline compliance & $>1$ million & $>1$ million & Dominant & Dominant \\
\hline 2.0 times baseline compliance & $>1$ million & Dominant & Dominant & Dominant \\
\hline
\end{tabular}

NOTE. The designation "> 1 million" refers to the model's calculated incremental cost-effectiveness ratio; the value is greater than $\$ 1$ million, which is much greater than the $\$ 50,000$ cost-effectiveness threshold. The designation "dominant" signifies that the distribution of preoperative bathing kits is dominant over the no-bath strategy; it is both less costly and more effective than no preoperative bath. SSI, surgical site infection.

${ }^{a}$ Baseline compliance refers to a mean compliance value of $15.3 \%$ (range, $8.23 \%-20.0 \%$ ). 
TABLE 3

Model Predictions of the Cost-Effectiveness of Chlorhexidine Baths on the Basis of Excess Length of Stay (LOS) Attributable to Surgical Site Infection (SSI)

\begin{tabular}{|c|c|c|c|c|}
\hline \multirow[b]{2}{*}{ SSI-attributable excess LOS, compliance rate ${ }^{a}$} & \multicolumn{4}{|c|}{ Bath efficacy in preventing SSIs } \\
\hline & $10 \%$ & $25 \%$ & $\mathbf{5 0 \%}$ & $\mathbf{7 5 \%}$ \\
\hline \multicolumn{5}{|l|}{5 days } \\
\hline One-fourth of baseline compliance & $>1$ million & $>1$ million & $>1$ million & $>1$ million \\
\hline One-half of baseline compliance & $>1$ million & $>1$ million & Dominant & Dominant \\
\hline Three-fourths of baseline compliance & $>1$ million & $>1$ million & Dominant & Dominant \\
\hline Baseline compliance & $>1$ million & $>1$ million & Dominant & Dominant \\
\hline 1.5 times baseline compliance & $>1$ million & Dominant & Dominant & Dominant \\
\hline 2.0 times baseline compliance & Dominant & Dominant & Dominant & Dominant \\
\hline \multicolumn{5}{|l|}{ Baseline LOS days } \\
\hline One-fourth of baseline compliance & $>1$ million & $>1$ million & $>1$ million & $>1$ million \\
\hline One-half of baseline compliance & $>1$ million & $>1$ million & Dominant & Dominant \\
\hline Three-fourths of baseline compliance & $>1$ million & Dominant & Dominant & Dominant \\
\hline Baseline compliance & $>1$ million & Dominant & Dominant & Dominant \\
\hline 1.5 times baseline compliance & Dominant & Dominant & Dominant & Dominant \\
\hline 2.0 times baseline compliance & Dominant & Dominant & Dominant & Dominant \\
\hline \multicolumn{5}{|l|}{15 days } \\
\hline One-fourth of baseline compliance & $>1$ million & Dominant & Dominant & Dominant \\
\hline One-half of baseline compliance & $>1$ million & Dominant & Dominant & Dominant \\
\hline Three-fourths of baseline compliance & Dominant & Dominant & Dominant & Dominant \\
\hline Baseline compliance & Dominant & Dominant & Dominant & Dominant \\
\hline 1.5 times baseline compliance & Dominant & Dominant & Dominant & Dominant \\
\hline 2.0 times baseline compliance & Dominant & Dominant & Dominant & Dominant \\
\hline
\end{tabular}

NOTE. The designation " >1 million" refers to the model's calculated incremental cost-effectiveness ratio; the value is greater than $\$ 1$ million, which is much greater than the $\$ 50,000$ cost-effectiveness threshold. The designation "dominant" signifies that the distribution of preoperative bathing kits is dominant over the no-bath strategy; it is both less costly and more effective than no preoperative bath.

${ }^{a}$ Baseline compliance refers to a mean compliance value of $15.3 \%$ (range, $8.23 \%-20.0 \%$ ). 
TABLE 4

Model Predictions of the Number of Bathing Kits That Need to be Dispensed to Preoperative Patients to Prevent 1 Surgical Site Infection

\begin{tabular}{lrrrr}
\hline & \multicolumn{4}{c}{ Bath efficacy } \\
\cline { 2 - 5 } Compliance rate $\boldsymbol{a}$ & $\mathbf{1 0 \%}$ & $\mathbf{2 5 \%}$ & $\mathbf{5 0 \%}$ & $\mathbf{7 5 \%}$ \\
\hline One-fourth of baseline compliance & 7,042 & 2,434 & 2,370 & 1,201 \\
One-half of baseline compliance & 4,220 & 2,198 & 817 & 499 \\
Three-fourths of baseline compliance & 2,273 & 1,311 & 579 & 364 \\
Baseline compliance & 1,191 & 780 & 443 & 269 \\
1.5 times baseline compliance & 1,101 & 700 & 269 & 185 \\
2.0 times baseline compliance & 815 & 364 & 212 & 137 \\
\hline
\end{tabular}

${ }^{a}$ Baseline compliance refers to a mean compliance value of $15.3 \%$ (range, $8.23 \%-20.0 \%$ ). 